ARTICLE

\title{
mTORC2 in the dorsomedial striatum of mice contributes to alcohol-dependent F-Actin polymerization, structural modifications, and consumption
}

\author{
Sophie Laguesse ${ }^{1,2}$, Nadege Morisot ${ }^{1,3}$, Khanhky Phamluong ${ }^{1}$, Samuel A. Sakhai ${ }^{1}$ and Dorit Ron ${ }^{1}$
}

\begin{abstract}
Actin is highly enriched at dendritic spines, and actin remodeling plays an essential role in structural plasticity. The mammalian target of rapamycin complex 2 (mTORC2) is a regulator of actin polymerization. Here, we report that alcohol consumption increases F-actin content in the dorsomedial striatum (DMS) of mice, thereby altering dendritic spine morphology in a mechanism that requires mTORC2. Specifically, we found that excessive alcohol consumption increases mTORC2 activity in the DMS, and that knockdown of Rictor, an essential component of mTORC2 signaling, reduces actin polymerization, and attenuates the alcoholdependent alterations in spine head size and the number of mushroom spines. Finally, we show that knockdown of Rictor in the DMS reduces alcohol consumption, whereas intra-DMS infusion of the mTORC2 activator, A-443654, increases alcohol intake. Together, these results suggest that mTORC2 in the DMS facilitates the formation of F-actin, which in turn induces changes in spine structure to promote and/or maintain excessive alcohol intake.
\end{abstract}

Neuropsychopharmacology (2018) 43:1539-1547; https://doi.org/10.1038/s41386-018-0012-1

\section{INTRODUCTION}

Actin cytoskeleton, the major component of dendritic spines [1], plays a major role in structural and functional synaptic plasticity [1, 2]. Strengthening or weakening of synaptic strength depends on actin cytoskeleton dynamics which is the driving force of spine morphology remodeling $[1,2]$. F-actin polymerization promotes spine enlargement and stabilization, whereas spine shrinkage requires F-actin disassembly $[2,3]$. Aberrant dendritic spine remodeling has been associated with psychiatric disorders and addiction $[4,5]$.

The mammalian target of rapamycin (mTOR) is a serine and threonine kinase [6]. When mTOR is in a complex with the adaptor protein Rictor, it is defined as mTOR complex 2 (mTORC2) [6, 7]. mTORC2 is activated by growth factors and leptin $[6,8]$, and by interacting with ribosomes [9]. mTORC2 phosphorylates substrates including AKT, serum, and glucocorticoid-induced protein kinase 1 (SGK1) and protein kinase C a (PKCa) [9-11].

mTORC2 plays an important role in actin polymerization $[8,12]$, and in the central nervous system (CNS), mTORC2 contributes to F-actin assembly in the hippocampus and to learning and memory $[13,14]$. Moreover, mTORC2 contributes the size and shape of cerebellar purkinje cells [15], and in the striatum, mTORC2 negatively regulates dopamine D2 receptor (D2R) surface expression and signaling [16]. Finally, dysregulation of mTORC2 function is linked to psychiatric disorders [17] such as schizophrenia [16], and morphine dependence [18], and, age-dependent memory loss is associated with a reduced mTORC2 activity in mice and in flies [14].
We previously reported that excessive alcohol consumption in mice induces structural plasticity in the dorsomedial striatum (DMS) [19], a brain region important for goal-directed behaviors [20]. Specifically, we found that repeated cycles of binge drinking and withdrawal increases the complexity of dendritic branches as well as the density of mushroom-shaped spines in medium spiny neurons (MSNs) in the DMS [19]. We recently observed that excessive alcohol intake increases the phosphorylation of AKT at the mTORC2 phosphorylation site, Serine 473 ( $\operatorname{Ser}^{473} \mathrm{AKT}$ ) in the DMS of mice [21] raising the possibility that $\mathrm{mTORC2}$ may be activated by alcohol in this brain region [21]. Given the fact that mTORC2 plays an important role in F-actin assembly, we hypothesized and found that alcohol alters dendritic spine morphology in the DMS via mTORC2-dependent actin cytoskeleton remodeling. We further show that mTORC2 in the DMS participates in mechanisms underlying alcohol drinking.

\section{MATERIALS AND METHODS}

Detailed information regarding reagents, animals and preparation of solutions can be found in supplementary material.

Western blot analysis

Western blot analysis was conducted as described in ref. [21]. Further information can be found in the supplementary material.

\footnotetext{
Department of Neurology, University of California, San Francisco, San Francisco, CA 94143, USA

Correspondence: Dorit Ron (dorit.ron@ucsf.edu)

${ }^{2}$ Present address: N.M. Brains On-line LLC, South San Francisco, CA, USA

${ }^{3}$ Present address: S.L. GIGA-Neurosciences, University of Liège, Liège, Belgium

The first two authors contributed equally to this work.
}

Received: 16 November 2017 Revised: 22 December 2017 Accepted: 5 January 2018

Published online: 7 February 2018 


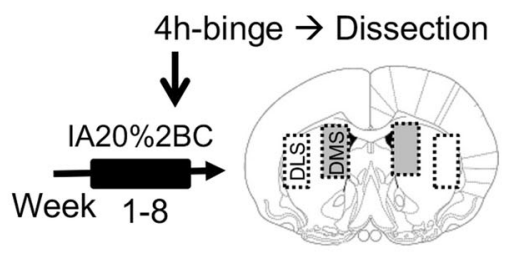

B

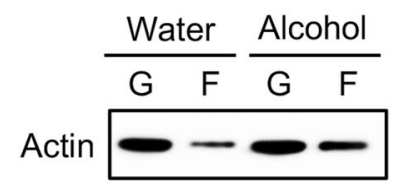

C

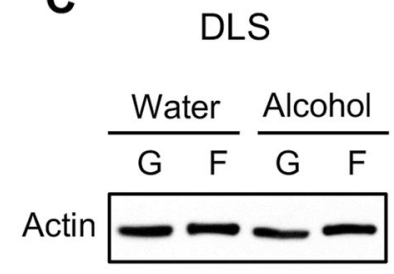

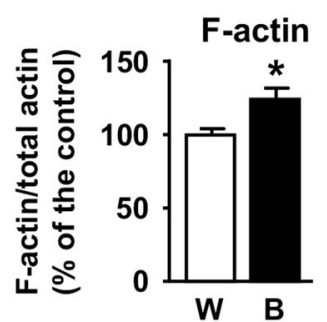
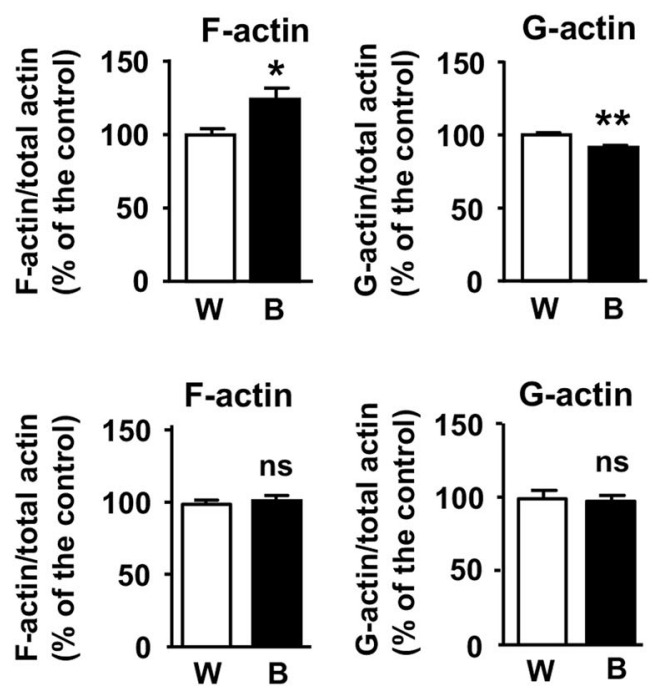

Fig. 1 Binge drinking of alcohol increases F-actin content in the DMS. Mice underwent IA20\%-2BC for 8 weeks. The DMS and DLS were dissected at the end of the last $4 \mathrm{~h}$-binge (B, black) alcohol drinking session. Control animals had access to water only (W, white). a Schematic representation of the experimental timeline, and DMS and DLS dissection. b, c Filamentous (F-) and globular (G-) actin content in the DMS (b) and DLS (c) were determined by western blot analysis. Data are presented as the mean ratio of F-actin or G-actin/total (F + G) actin \pm S.E.M and expressed as percentage of water control. b DMS: F-actin $t_{(12)}=2.79, p=0.016$; G-actin $t_{(12)}=3.66 p=0.003 ; n=7$ water, alcohol. c DLS: F-actin $t_{(14)}=0.35, p=0.734 ; \mathrm{G}$-actin $t_{(14)}=0.48 p=0.637 ; n=8$ water, alcohol. ${ }^{*} p<0.05,{ }^{* *} p<0.005$

F-actin/G-actin assay

F-actin/G-actin assay was performed using the G-actin/F-actin kit (Cytoskeleton Inc.) as previously described in ref. [22]. Further information can be found in the supplementary material.

\section{Immunohistochemistry}

Immunohistochemistry was conducted as described previously [23]. Further information can be found in the supplementary material.

Generation of a lentivirus-expressing shRNA targeting Rictor The 19 nucleotide short hairpin RNA (shRNA) sequence targeting Rictor 5'-GCGAGCTGATGTAGAATTA-3' was selected using siRNA Wizard v3.1 (InvivoGen, San Diego, CA). The scrambled (SCR) 19 nucleotide sequence $5^{\prime}$-GCGCUUAGCUGUAGGAUUC-3' was used as control. The generation of a lentivirus (Ltv) expressing shRictor or SCR control was conducted as described in ref. [22]. Intra-DMS infusion of lentivirus was conducted as described in ref. [23]. Further information regarding the preparation, characterization and usage of the virus can be found in the supplementary material.

Dendritic branches and spine analysis

Morphological analysis was conducted as described previously [22]. Further information can be found in the supplementary material.

\section{Behavioral paradigms}

Intermittent access to $20 \%$ alcohol (IA20\%-2BC), or $0.01 \%$ saccharin (IA0.01\%sac) are described in [21], and locomotor testing is described in ref. [23]. Further details can be found in the supplementary material.
Microinfusion of the mTORC2 activator A-443654

Mice received an intra-DMS administration of vehicle (0.1\% DMSO in PBS) or A-443654 [24] $(1 \mu \mathrm{g} / \mu \mathrm{l} ; 1 \mu \mathrm{l} / \mathrm{side}) 15 \mathrm{~min}$ prior to an alcohol drinking session. Each mouse received each treatment (vehicle and/or A-443654) twice on different test sessions, in a counter-balanced manner. Further details regarding coordinates of cannula placement and experimental timeline are described in the supplementary material.

Data analysis

Data are expressed as mean \pm S.E.M. Biochemical data were analyzed using unpaired $t$ test or two-way ANOVA, followed by post hoc Turkey's test, as specified in the Figure Legends. Behavioral data were analyzed using paired or unpaired $t$ test as well as one-way analysis of variance with repeated measures (RMANOVA), as specified in the Figure Legends. Significant main effects or interaction of the RM-ANOVA were further investigated with Turkey's post hoc testing. Statistical significance was set at $p<0.05$.

\section{RESULTS}

Alcohol drinking increases F-actin content in the dorsomedial striatum

We previously reported that excessive alcohol consumption induces remodeling of dendritic branches and spines in the DMS of mice [19]. Actin is the main component of dendritic spines, and is a critical regulator of dendritic spines structure [25]. Therefore, we first tested whether alcohol intake modifies the ratio between globular actin monomers (G-actin) and filamentous actin (F-actin). To do so, mice underwent 8 weeks of IA20\%-2BC, the DMS was collected $4 \mathrm{~h}$ after the beginning of the last alcohol 
A

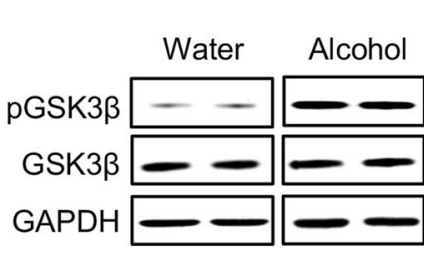

C

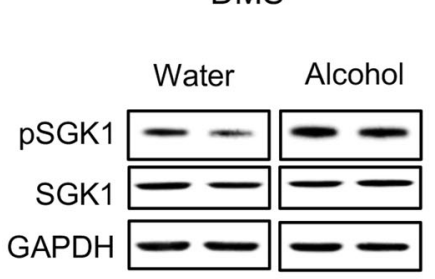

PSK3 $\beta$

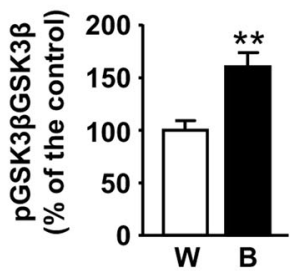

PSK1

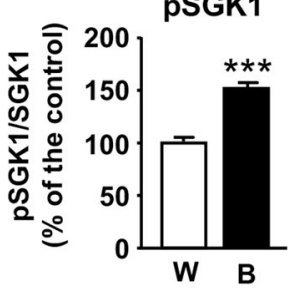

B

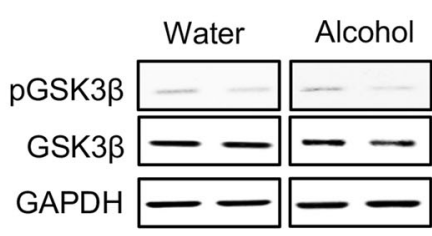

D

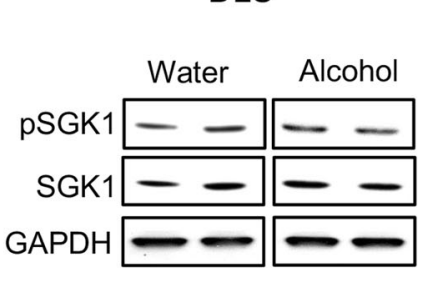

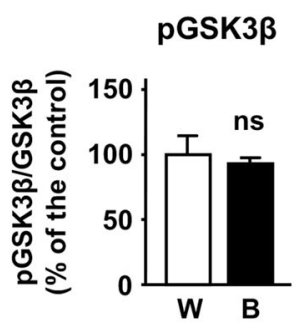

Fig. 2 Binge drinking of alcohol activates mTORC2 in the DMS. Mice drinking paradigm and dissection scheme are outlined in Fig. 1a. The DMS and DLS were dissected at the end of the last $4 \mathrm{~h}$-binge ( $\mathrm{B}$, black) alcohol drinking session. Control animals had access to water only (W, white). a, b Ser ${ }^{9}$ GSK3 $\beta$ phosphorylation (pGSK3 $\beta$ ) was measured by western blot analysis. GAPDH immunoreactivity was used as an internal loading control. Data are presented as the mean ratio of Ser ${ }^{9} \mathrm{GSK} 3 \beta$ to total GSK3 $\beta \pm$ S.E.M, and expressed as percentage of water control. a DMS: $t_{(15)}=3.87, p=0.0015 ; n=8$ water, 9 alcohol. b DLS: $t_{(7)}=0.81, p=0.444 ; n=4$ water, 5 alcohol. c $_{\text {, }} \mathbf{d}$ Ser ${ }^{422}$ SGK1 phosphorylation (pSGK1) was measured by western blot analysis. GAPDH immunoreactivity was used as an internal loading control. Data are presented as the mean ratio of Ser ${ }^{422}$ SGK1 to total SGK1 \pm S.E.M, and expressed as percentage of water control. c DMS: $t_{(15)}=4.87, p=0.0002 ; n=8$ water, 9 alcohol. d DLS: $t_{(15)}=0.31, p=0.762 ; n=8$ water, 9 alcohol. ${ }^{* *} p<0.01$, ${ }^{* * *} p<0.001$

drinking session, and G-actin and F-actin content were evaluated (Fig. 1a; Table S1). We found that repeated cycles of excessive alcohol drinking and withdrawal significantly increase the F-actin content and reduce the G-actin content in the DMS (Fig. 1b). However, no change in F- and G-actin content was observed in the neighboring striatal region, the dorsolateral striatum (DLS) (Fig. 1c). These data suggest that excessive alcohol consumption increases F-actin assembly specifically in the DMS.

mTORC2's activity is increased in the DMS of mice with a history of excessive alcohol drinking and withdrawal

One of mTORC2's well-characterized substrates is the serine and threonine kinase, AKT [11]. Phosphorylation of Ser ${ }^{473}$ AKT by mTORC2 is required for the full activation of the kinase [26]. We found that Ser ${ }^{473}$ AKT phosphorylation was elevated in the DMS [21]. Therefore, to test if AKT is indeed fully activated in the DMS, we measured the phosphorylation of its substrate GSK3 $\beta$ [26]. As shown in Fig. 2a, alcohol intake increased GSK3 $\beta$ phosphorylation in the DMS. In contrast, GSK3 $\beta$ phosphorylation was unaltered in the DLS (Fig. 2b). These results are in accordance with our previous results showing no phosphorylation of $\mathrm{Ser}^{473} \mathrm{AKT}$ in this brain region [21]. These data suggest that $\mathrm{MTORC2}$ is activated by alcohol in the DMS.

To confirm that mTORC2 is indeed activated by alcohol, we measured the phosphorylation levels of another mTORC2 substrate, serum and glucocorticoid-induced protein kinase 1 (SGK1), which is phosphorylated by mTORC2 at the Serine 422 residue (Ser ${ }^{422}$ SGK1) [10]. We found that alcohol intake increased SGK1 phosphorylation in the DMS but not the DLS (Fig. 2c-d). Together, these results indicate that mTORC2 is activated specifically in the DMS of mice that consumed alcohol.

Knockdown of rictor in the DMS reduces mTORC2 activity and actin polymerization

The adaptor protein Rictor is essential for the activity of mTORC2 [12], and Rictor manipulation has been used to study mTORC2 function $[13,16]$. We hypothesized that mTORC2 in the DMS contributes to the formation of actin filaments. To examine this possibility, we examined consequence of mTORC2 inhibition on actin dynamics by knocking down Rictor in DMS medium spiny neurons (MSNs). To do so, we generated a lentivirus-expressing green fluorescence protein (GFP) and a short hairpin RNA (shRNA) sequence targeting Rictor (Itv-shRictor), or a scrambled sequence (Itv-SCR) as a control. High level of Itv-shRictor infection in the DMS MSNs was observed 4 weeks after virus administration as shown by co-staining of GFP with the neuronal marker NeuN (Fig. 3a). Infection of DMS neurons with Itv-shRictor produced a significant knockdown of the protein compared to mice infected with the ItvSCR control (Fig. 3b). We then confirmed that downregulation of Rictor impairs mTORC2 activity by showing that the basal phosphorylation level of $\mathrm{Ser}^{473} \mathrm{AKT}$ was reduced in the DMS of Itv-shRictor-infected mice as compared to mice infected with the Itv-SCR (Fig. 3b). Importantly, as shown in Fig. 3c, knockdown of Rictor in the DMS produced a significant reduction in F-actin content, and a corresponding increase in G-actin content suggesting that $\mathrm{mTORC2}$ in the DMS participates in the assembly of F-actin.

Alcohol-dependent alterations of dendritic spines structure depend on mTORC2

We previously reported that excessive alcohol consumption increases the length and complexity of dendritic branches, as well as the density of mushroom-shaped spines in DMS MSNs [19]. Dendritic complexity and spine enlargement depend on the formation of actin filaments [1]. As mTORC2 plays a role in F-actin assembly in the hippocampus [12, 13], and in the DMS (Fig. 3C), we hypothesized that the changes in actin dynamics triggered by alcohol produce remodeling of dendritic structure through a mechanism that depends on mTORC2. To test this hypothesis, mice underwent 4 weeks of IA20\%-2BC (Table S2), and were then infused bilaterally with a low titer $\left(1 \times 10^{5} \mathrm{pg} / \mathrm{ml}\right)$ of Itv-shRictor or Itv-SCR in the DMS. One week after surgery, mice had access to 4 more weeks of IA20\%-2BC (Fig. 4a). Low titer infection allowed the labeling of sparse number of neurons, ensuring the analysis of dendritic branches (Fig. 4b), and dendritic spines (Fig. 4h). 
A
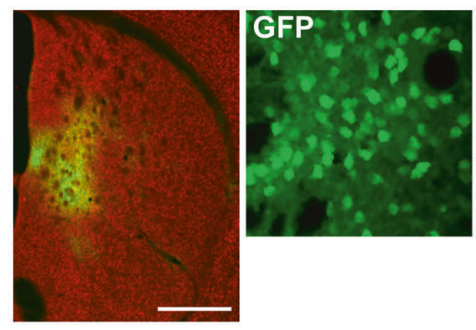

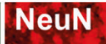

B

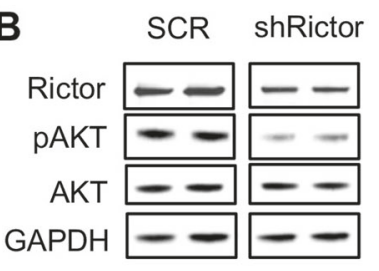

C

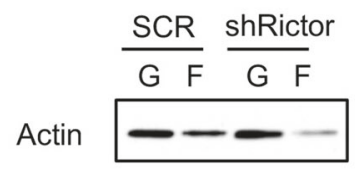

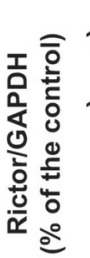

Rictor

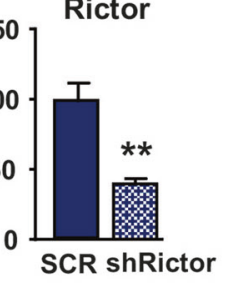

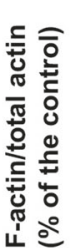

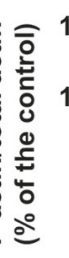

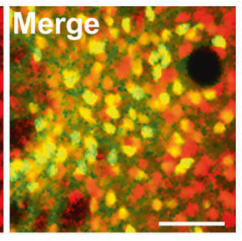
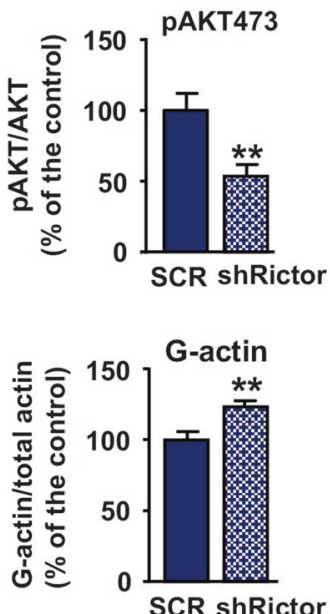

Fig. 3 Downregulation of mTORC2 in the DMS reduces F-actin content. Lentivirus (Itv)-expressing shRictor or SCR and GFP reporter was bilaterally infused into the DMS. Striatal tissues were collected 4 weeks after virus infusion and used for IHC (a) or western blot (b, c) analysis. a DMS-containing slices were co-stained with anti-GFP and NeuN antibodies. Left image ( $\times 5$ magnification) depicts the specificity of the infection site; scale bar $1 \mathrm{~mm}$. Right images ( $\times 20$ magnification) depicts DMS-infected neurons; scale bar $40 \mu \mathrm{m}$. $\mathbf{b}$ Levels of Rictor, AKT and Ser ${ }^{43}$ AKT phosphorylation (pAKT) were measured by western blot analysis. GAPDH immunoreactivity was used as an internal loading control. Data are presented as the mean ratio of Rictor/GAPDH or pAKT/AKT \pm S.E.M. b Rictor knockdown $t_{(12)}=4.10, p=0.0015$. b Ser ${ }^{473}$ AKT phosphorylation $t_{(12)}=3.19, p=0.008, n=7$ per virus treatment. c The filamentous $(\mathrm{F})$ and globular $(\mathrm{G})$ actin contents were determined by western blot analysis. Data are presented as the mean ratio of F-actin or G-actin /total $(\mathrm{F}+\mathrm{G})$ actin \pm S.E.M, and expressed as the percentage of Itv-SCR. F-actin, $t_{(9)}=3.36, p=0.0084 ; \mathrm{G}$-actin, $t_{(9)}=3.4, p=0.008 . n=5-6$ per virus treatment; ${ }^{* *} p<0.01$

Importantly, low titer infection did not alter the amount of alcohol consumed by shRictor-infected mice as compared to Itv-SCRinfected controls, ensuring that both groups of mice received similar amounts of alcohol (Table S2). Replicating our previous findings [19], we found that excessive alcohol consumption increased the length and complexity of the dendritic branches of DMS MSNs (Fig. 4c-g). Sholl analysis revealed that dendrites 50-120 $\mu \mathrm{m}$ away from the soma exhibited more intersections in the alcohol-consuming Itv-SCR mice as compared with water only drinking Itv-SCR mice (Fig. 4c,d). Furthermore, the total dendritic length (Fig. 4e), as well as the number of branches (Fig. 4f), and end points (Fig. 4g) were also increased. Rictor knockdown did not alter the complexity or the length of dendritic branches (Fig. 4c-g), suggesting that alcohol-dependent alterations in the dendritic branches do not require mTORC2.

Next, we examined the density, length and morphology of dendritic spines of DMS MSNs. Figure $4 \mathrm{~h}$ shows a representative image of a distal (3rd or 4th order) dendritic branch bearing spines in the four conditions (Water/Itv-SCR, Water/ltv-shRictor, Alcohol/ Itv-SCR, Alcohol/Itv-shRictor). Spine density was not affected by either alcohol or Rictor knockdown (Fig. $4 \mathrm{~m}$ ). In line with our previous findings [19], alcohol consumption significantly increased the width of the spine head (Fig. 4i) and spine area (Fig. 4j), and concomitantly decreased the spine length-to-width ratio (Fig. 4k), without changing spine length (Fig. 4l). Knockdown of Rictor in the water consuming mice significantly reduced the spine head width (Fig. 4i), while increasing the length-to-width ratio (Fig. 4k), and the spine length (Fig. 4l). These changes were not the result of different levels of GFP in dendritic spines, as GFP intensities in the parent dendritic branch were identical across groups (Fig. 4n). Importantly, knockdown of Rictor in alcohol-consuming mice rescued the alcohol-dependent phenotype in spine head width, length to width ratio and spine area (Fig. $4 \mathrm{i}-\mathrm{k}$ ). Together, these data suggest that the alcohol-dependent neuroadaptations of dendritic spines in the DMS are mediated by mTORC2.

Dendritic spines can be classified into four subclasses: filopodia, thin, stubby, and mushroom types [27]. Mushroom spines are considered mature, while thin spines are immature [27]. We found that alcohol intake significantly increased the number of mushroom-shaped spines at the expense of thin and filopodia spines, and that downregulation of $\mathrm{mTORC2}$ in the DMS resulted in the opposite pattern (i.e., an increased proportion of filopodia and thin spines, with a decrease in the number of mushroom spines) (Fig. 4o). Importantly, the alcohol-dependent alterations of spines were not observed in the alcohol drinking mice infected with ItvshRictor (Fig. 40). These results suggest that $\mathrm{mTORC2}$, by regulating actin dynamics, contributes to spine size enlargement and the stabilization of mature mushrooms-shaped spines. Our results further suggest that the dendritic spine remodeling induced by alcohol in the DMS is mediated, at least in part, by mTORC2.

Inhibition of mTORC2 function in the DMS reduces alcohol intake Next, we reasoned that the mTORC2-dependent structural alterations in dendritic spines contribute to the neuroadaptations underlying the development and/or maintenance of alcohol consumption. Therefore, we tested whether knockdown of Rictor in the DMS affects alcohol intake. Four weeks after bilateral infusion of Itv-SCR or Itv-shRictor in the DMS, mice underwent 
A

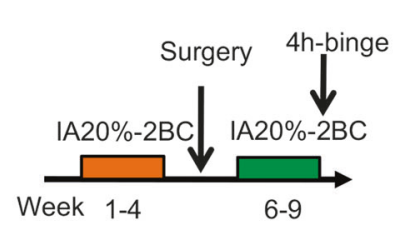

B

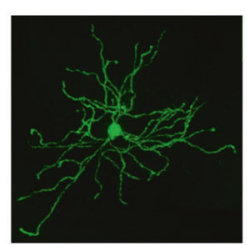

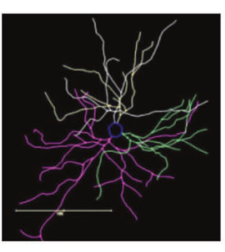

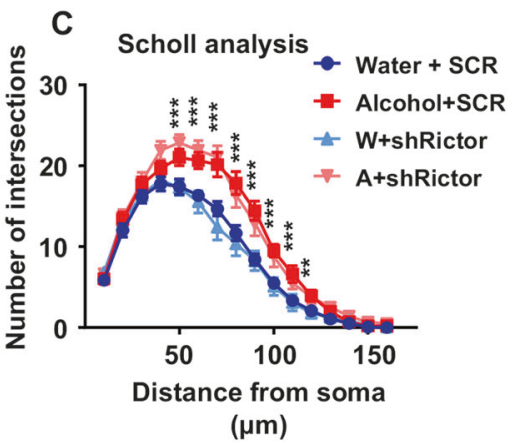

G
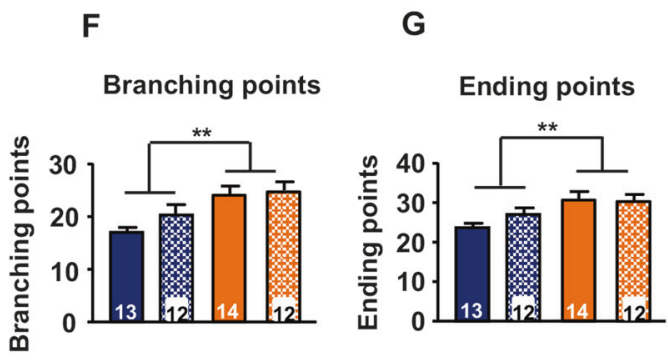

W+SCR

W+shRictor

$\square$ A+SCR

A+shRictor
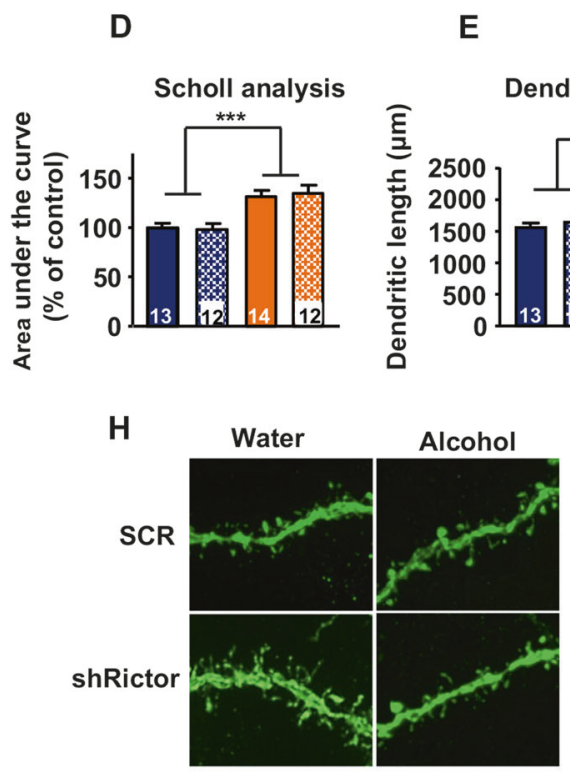

K

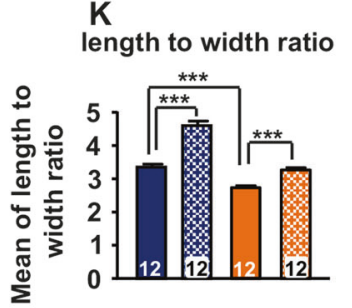

E

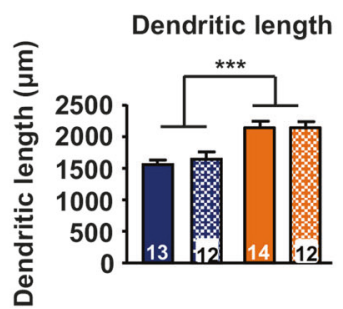

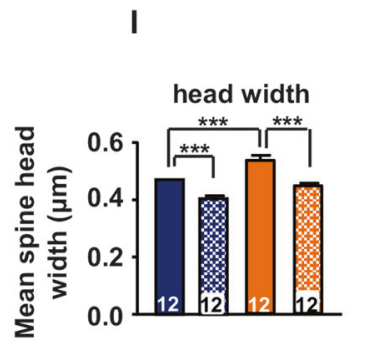

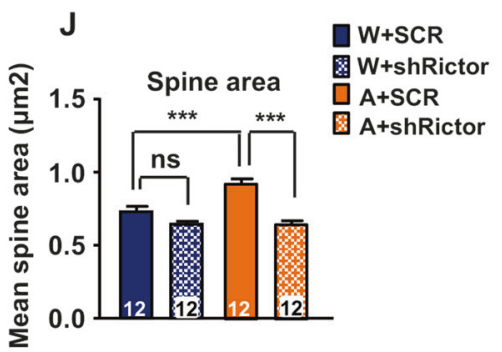

L Spine length
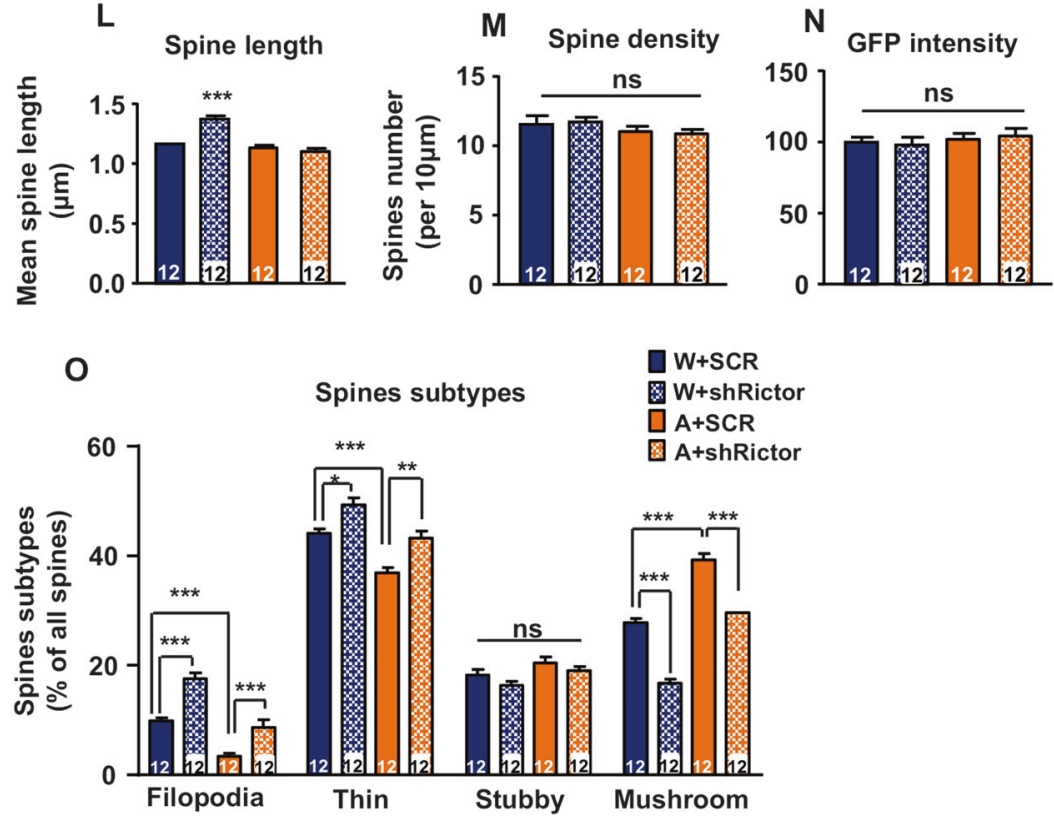

IA20\%-2BC (Fig. 5a), and alcohol intake was measured 4 and $24 \mathrm{~h}$ after the beginning of each drinking session. As shown in Fig. 5b, knockdown of Rictor in the DMS significantly reduced binge-like alcohol intake across sessions without affecting water intake (Fig. 5c). When measured at the end of the $24 \mathrm{~h}$ drinking session, mice infected with Itv-shRictor or Itv-SCR drank similar levels of alcohol $\left(t_{(13)}=0.49, p=0.63\right.$, data not shown), and water $\left(t_{(13)}=0.21, p=0.83\right.$, data not shown). Importantly, knockdown of Rictor in the DMS did not affect the consumption of the rewarding substance saccharin, nor did it alter locomotor behavior 
Fig. 4 Alcohol regulates MSN dendritic spine morphology via mTORC2. Mice underwent 4 weeks of IA20\%-2BC. Mice consuming water only were used as control. Low titer of Ltv-shRictor or Itv-SCR $\left(1 \times 10^{5} \mathrm{pg} / \mathrm{ml}\right)$ was infused bilaterally into the DMS, and after 1 week of recovery, mice were given 4 more weeks of IA20\%-2BC access. Four hours after the beginning of the last drinking session, mice were perfused, and MSNs morphology was analyzed. a Schematic representation of the experiment timeline. $\mathbf{b}$ Left, sample image of a GFP-positive DMS MSN; Right, semi-automated two-dimensional reconstruction of the GFP-positive neuron. Scale bar, $100 \mu \mathrm{m}$. c, d Analysis of neuronal dendritic arborization. Sholl analysis was performed on reconstructed neurons and the area under the curve was calculated. Two-way ANOVA showed a significant main effect of alcohol $\left(\mathrm{F}_{(1,46)}=27.24, p<0.001\right)$ but no effect of virus $\left(\mathrm{F}_{(1,46)}=0.007, p=0.935\right)$ and no interaction $\left(\mathrm{F}_{(1,46)}=0.17, p=\right.$ 0.683 ) between the four conditions (water/SCR (blue), water/shRictor (hatched blue), alcohol/SCR (orange), alcohol/shRictor (hatched orange). The number of intersections was analyzed for each point, and two-way ANOVA showed a significant main effect of alcohol at 50, 60, 70, 80, 90, 110 , and $120 \mu \mathrm{m}$ from the soma, but no effect of virus or interaction. e Total dendritic length. Data are presented as the average total dendritic length \pm S.E.M and expressed in $\mu \mathrm{m}$. Two-way ANOVA showed a significant main effect of alcohol $\left(\mathrm{F}_{(1,47)}=26.35, p<0.001\right)$ but no effect of virus $\left(F_{(1,47)}=0.16, p=0.69\right)$ and no interaction $\left(F_{(1,47)}=0.18, p=0.677\right)$. $\mathbf{f}$ Number of branching points. Data are presented as the average number of branching points \pm S.E.M. Two-way ANOVA showed a significant main effect of alcohol $\left(\mathrm{F}_{(1,47)}=11.98, p=0.0012\right)$ but no effect of virus $\left(F_{(1,47)}=2.81, p=0.1\right)$, and no interaction $\left(F_{(1,47)}=1.51, p=0.225\right)$. g Number of ending points. Data are presented as the average number of ending points \pm S.E.M. Two-way ANOVA showed a significant main effect of alcohol $\left(\mathrm{F}_{(1,47)}=10.26, p=0.0024\right)$ but no effect of virus $\left(\mathrm{F}_{(1,47)}\right.$ $=0.81, p=0.372)$ and no interaction $\left(\mathrm{F}_{(1,47)}=1.19, p=0.28\right)$. $\mathbf{h}$ Representative $\mathrm{x} 100$ confocal z-stack images of dendritic segments bearing spines for the four experimental conditions (water/SCR, water/shRictor, alcohol/SCR, alcohol/shRictor). Scale bar, $5 \mu \mathrm{m}$. i Average spine head width. Data are presented as the average of spine head width \pm S.E.M and are expressed in $\mu \mathrm{m}$. Two-way ANOVA showed a significant main effect of alcohol $\left(\mathrm{F}_{(1,44)}=24.43, p<0.001\right)$ and virus $\left(\mathrm{F}_{(1,44)}=49.16, p<0.001\right)$ but no interaction $\left(\mathrm{F}_{(1,44)}=1.03, p=0.316\right)$. post hoc Turkey's test detected a significant difference between water and alcohol within the SCR group $(p<0.001)$, a significant difference between SCR and shRictor within the water group $(p<0.001)$ and within the alcohol group $(p<0.001)$. $\mathbf{j}$ Spine area of DMS MSNs. Data are presented as the average spine area \pm S.E.M and are expressed in $\mu \mathrm{m}^{2}$. Two-way ANOVA showed a significant main effect of alcohol $\left(\mathrm{F}_{(1,44)}=8.94, p=0.005\right)$ and virus $\left(F_{(1,44)}=33.9, p<0.001\right)$, and a significant interaction $\left(F_{(1,44)}=9.27, p=0.004\right)$. Post hoc Turkey's test detected a significant difference between water and alcohol within the SCR group $(p<0.001)$, a significant difference between SCR and shRictor within the alcohol group $(p<$ $0.001)$ but no difference between SCR and shRictor within the water group $(p=0.217)$. $\mathbf{k}$ Average length to width ratio of DMS MSNs. Data are presented as the average of spine length to width ratio \pm S.E.M. Two-way ANOVA showed a significant main effect of alcohol $\left(\mathrm{F}_{(1,44)}=111.6, p\right.$ $<0.001)$ and virus $\left(\mathrm{F}_{(1,44)}=93.83, p<0.001\right)$, and a significant interaction $\left(\mathrm{F}_{(1,44)}=14.75, p<0.001\right)$. Post hoc Turkey's test detected a significant difference between water and alcohol within the SCR group $(p<0.001)$, a significant difference between SCR and shRictor within the water group $(p<0.001)$ and within the alcohol group $(p<0.001)$. I Average spine length of DMS MSNs of mice. Data are presented as the average spine length \pm S.E.M and are expressed in $\mu \mathrm{m}$. Two-way ANOVA showed a significant main effect of alcohol $\left(\mathrm{F}_{(1,44)}=41.68, p<0.001\right)$ and virus $\left(F_{(1,44)}=13.86, p<0.001\right)$ and a significant interaction $\left(F_{(1,44)}=25.73, p<0.001\right)$. Post hoc Turkey's test detected a significant difference between SCR and shRictor within the water group $(p<0.001)$ but no difference within the alcohol group $(p=0.776)$ and no difference between water and alcohol in the SCR group $(p=0.763)$. $\mathbf{m}$ Spine density of DMS MSNs. Data are presented as the average spine density per $10 \mu \mathrm{m} \pm$ S.E.M. Two-way ANOVA showed no main effect of alcohol $\left(\mathrm{F}_{(1,44)}=2.55, p=0.118\right)$ or virus $\left(\mathrm{F}_{(1,44)}=0.001, p=0.973\right)$ and no interaction $\left(F_{(1,44)}=0.13, p=0.721\right) . \mathbf{n}$ GFP intensity. Data are presented as the average GFP intensity \pm S.E.M and are expressed as percentage of the water/ SCR control. Two-way ANOVA showed no main effect of alcohol $\left(\mathrm{F}_{(1,44)}=0.0004, p=0.995\right)$ or virus $\left(\mathrm{F}_{(1,44)}=0.89, p=0.349\right)$ and no interaction $\left(F_{(1,44)}=0.23, p=0.631\right.$. o Percentage of filopodia, thin, stubby, and mushroom-type spines of DMS MSNs. Data are presented as the average \% of each type of spines across the four conditions \pm S.E.M. Filopodia-type spines, two-way ANOVA showed a significant main effect of alcohol $\left(\mathrm{F}_{(1,44)}=124.4, p<0.001\right)$ and virus $\left(\mathrm{F}_{(1,44)}=89, p<0.001\right)$ but no significant interaction $\left(\mathrm{F}_{(1,44)}=3.27, p=0.077\right)$. Post hoc Turkey's test detected a significant difference between water and alcohol in the SCR group $(p<0.001)$, and a significant difference between SCR and shRictor within the water group $(p<0.001)$, and within the alcohol group $(p<0.001)$; Thin spines, two-way ANOVA showed a significant main effect of alcohol $\left(\mathrm{F}_{(1,44)}=34, p<0.001\right)$ and virus $\left(\mathrm{F}_{(1,44)}=25.4, p<0.001\right)$ but no interaction $\left(\mathrm{F}_{(1,44)}=0.26, p=0.613\right)$. Post hoc Turkey's test detected a significant difference between water and alcohol within the SCR group $(p<0.001)$, and a significant difference between SCR and shRictor within the water group $(p=0.013)$ and the alcohol group $(p=0.0017)$; Stubby spines, two-way ANOVA showed no significant main effect of alcohol $\left(F_{(1,44)}=1.41, p=0.242\right)$ or virus $\left(F_{(1,44)}=1.190, p=0.282\right)$ and no interaction $\left(F_{(1,44)}=0.94, p=0.337\right)$; Mushroom spines, two-way ANOVA showed a significant main effect of alcohol $\left(\mathrm{F}_{(1,44)}=178.8, p<0.001\right)$ and virus $\left(\mathrm{F}_{(1.44)}=130.8, p<0.001\right)$, but no interaction $\left(\mathrm{F}_{(1.44)}=\right.$ $0.61, p=0.44)$. Post hoc Turkey's test detected a significant difference between water and alcohol within the SCR group ( $p<0.001)$, and a significant difference between SCR and shRictor within the water group $(p<0.001)$ and within the alcohol group $(p<0.001)$. $n=12-14$ neurons, 5 mice per condition. ${ }^{*} p<0.05,{ }^{* *} p<0.01,{ }^{* *} p<0.001$

(Figure S1). Together, these data reveal that mTORC2 in the DMS contributes to the development of binge-like alcohol drinking without altering general reward processing or spontaneous locomotor activity.

Infusion of the mTORC2 activator, A-445634, in the DMS of mice increases alcohol consumption

Finally, we reasoned that since downregulation of mTORC2 signaling in the DMS reduces excessive alcohol drinking, then activation of this pathway would promote alcohol consumption. To test this hypothesis, mice were implanted with a guide cannula targeting the DMS (Figure S2), and were then subjected to IA20\%-2BC for 3 weeks followed by a microinfusion of vehicle $(0.1 \%$ DMSO in PBS) or the mTORC2 activator, A-445634 $(1 \mu \mathrm{g} / \mu \mathrm{l})$ [24], $15 \mathrm{~min}$ before the beginning of an alcohol drinking session (Fig. 5d). Intra-DMS infusion of A-445634 produced an increase in alcohol consumption within the first hour of the session, as compared to vehicle-treated mice (Fig. 5e). In contrast, water consumption was not significantly altered by A-445634 administration although a non-significant $(p=0.254)$ trend toward an increase in water intake was observed (Fig. 5f). The increase in alcohol intake by A-445634 was no longer observed $2 \mathrm{~h}$ after the beginning of the test session $\left(t_{(8)}=0.27, p=0.80\right.$, data not shown). Importantly, the effect of A-445634 on alcohol intake was specific, as saccharin intake was unaltered following infusion of the drug into the DMS $1 \mathrm{~h}$ after the beginning of the test session (Figure S2). These results indicate that enhancing mTORC2 activity within the DMS exacerbates alcohol drinking without general changes in reward sensitivity.

\section{DISCUSSION}

Here, we present data to suggest that alcohol activates mTORC2 in the DMS leading to the formation of F-actin, which in turn increases spine head size and area, as well as the proportion of mature mushroom-shaped spines. We further show that mTORC2 in the DMS contributes to the development of excessive alcohol consumption.

Alcohol increases F-Actin assembly in the DMS

We show herein that excessive alcohol intake increases F-actin assembly in the DMS. Actin polymerization, which contributes to 


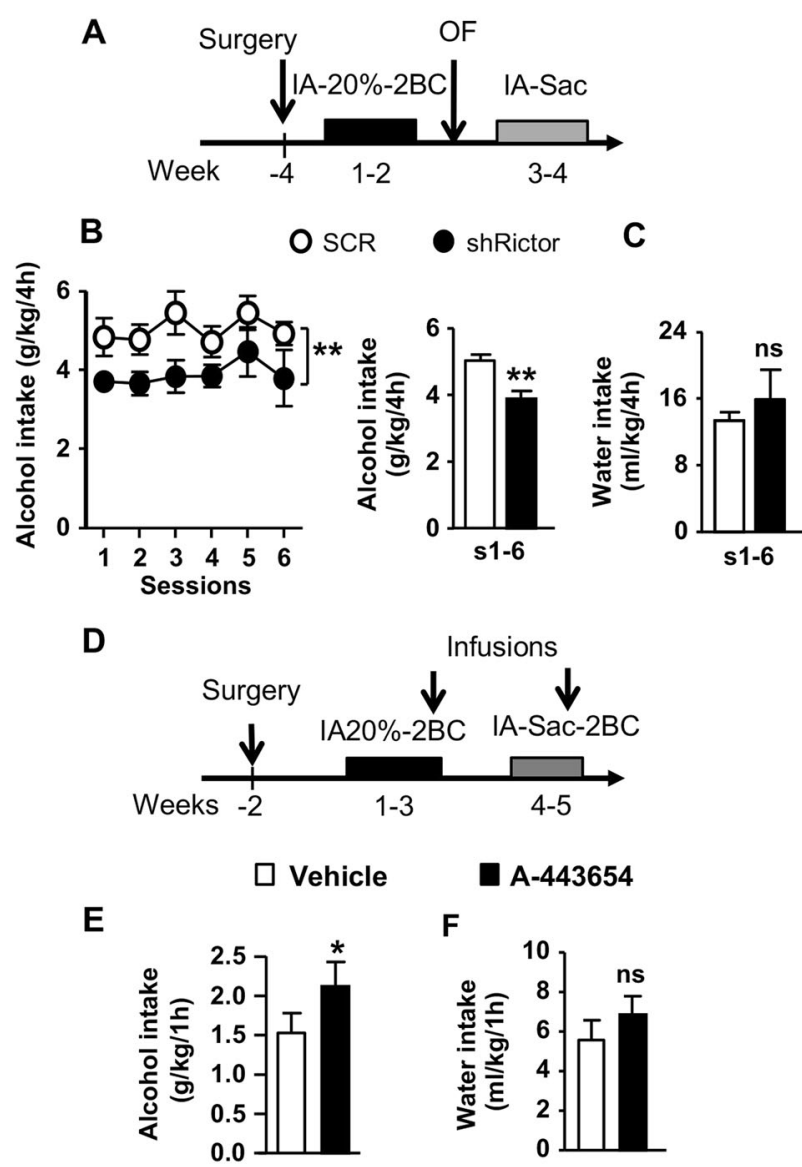

Fig. 5 Downregulation of $\mathrm{mTORC2}$ in the DMS reduces binge-like alcohol drinking whereas activation of mTORC2 promotes it. a Schematic representation of experimental timeline of experiment Mice were infused bilaterally with Itv-shRictor or Itv-SCR in the DMS. Four weeks later, animals underwent an IA20\%-2BC for 6 sessions. Following 2 weeks of alcohol deprivation in which mice had access to water only, they were subjected to an IA0.01\% sac for six sessions. Fluid intake was measured $4 \mathrm{~h}$ after the beginning of each drinking session. b, c Black circle and bar depicts Itv-shRictor and white circle and bar depicts Itv-SCR. b Alcohol intake $(\mathrm{g} / \mathrm{kg} / 4 \mathrm{~h})$ across drinking sessions (left, main effect of virus $\mathrm{F}_{(1,13)}=14.18, p=0.0024$ ) and averaged (sessions 1 to 6) alcohol intake (right, $t_{(13)}=3.77$, $p=0.0024)$. c Water $(\mathrm{ml} / \mathrm{kg} / 4 \mathrm{~h})$ intake on session 1 to 6 (s1-6) in the IA20\%-2BC paradigm $t_{(13)}=0.72, p=0.484$. d Schematic representation of timeline of experiment. Bilateral guide cannula were surgically implanted in the DMS. Two weeks later, mice underwent IA20\%-2BC for 3 weeks and Vehicle or A-443654 $(1 \mu \mathrm{g} / \mu \mathrm{l})$ was infused in the DMS $15 \mathrm{~min}$ before the beginning of a drinking session. Fluid intake was measured $1 \mathrm{~h}$ after the beginning of the drinking session. Following 2 weeks of access to water only, mice were subjected to IA $0.01 \%$ sac for 2 weeks prior to Vehicle or A-443654 $(1 \mu \mathrm{g} / \mu \mathrm{l})$ administration e Alcohol $(\mathrm{g} / \mathrm{kg} / 1 \mathrm{~h})$ intake following intra-DMS infusion of vehicle (white) or A-443654 (black) $t_{(8)}=2.38, p=0.0449$. f Water $(\mathrm{ml} / \mathrm{kg} / 1 \mathrm{~h})$ intake $t_{(8)}=1.23, p=0.254$. Data are expressed as mean \pm S.E.M. b-c $n=7-8$ per treatment, ${ }^{* *} p<0.01$ vs. Itv-SCR; e-f $n=9$ per treatment, ${ }^{*} p<0.05$ vs. vehicle

dendritic spines morphology [1], has been implicated as a target of alcohol and other drugs of abuse, as modifying actin dynamics can shape drugs of abuse-related behaviors [4]. For example, cocaine administration decreases the phosphorylation levels of cofilin, a regulator of actin filaments [28], and actin polymerization promotes morphine place preference and drives cocaine-induced reinstatement $[29,30]$. We recently reported that excessive alcohol consumption produce structural and synaptic alterations in the nucleus accumbens (NAc), which depend on actin remodeling
[22]. Together, these studies suggest that actin dynamics is a convergent mechanism shared by drugs of abuse.

Interestingly, although alcohol promotes actin polymerization in the NAc [22] and in the DMS, no change was detected in the DLS suggesting that alcohol's actions are restricted. Brain region and cell-type specificity in molecular adaptations induced by alcohol in the CNS have been gaining interest [46]. The potential explanation to the selectivity of alcohol's actions on F-actin assembly is unknown although it is likely to be due to the mechanism that promotes the formation of F-actin. Specifically, we provide evidence to suggest that mTORC2 is of upstream F-actin assembly in the DMS, and the formation of F-actin in the NAC depends on the activation of mTOR in complex 1 (mTORC1) [22]. However, neither mTORC2 nor mTORC1 is activated in the DLS $([21,22]$ and data herein). Furthermore, it is of interest to note that alcohol intake activates the same kinase (mTOR) in different complexes (mTORC2 and mTORC1), in two substriatal regions leading in both regions to the same outcome, e.g. actin polymerization and dendritic spine modifications. It is tempting to speculate that each arm of mTOR signaling in the striatum contribute to different behavioral outcomes that together drive the development and maintenance of AUD.

mTORC2 is activated by alcohol in the DMS

We found that alcohol activates mTORC2 in the DMS as indicated by the phosphorylation of the mTORC2 substrates, AKT [21], and SGK1. Furthermore, we show that the well-characterized AKT substrate, GSK3 $\beta$, is phosphorylated by alcohol in this brain region, data which are in line with a recent study by Cheng and colleagues [31]. The specific mechanism by which excessive alcohol drinking activates mTORC2 signaling in the DMS remains unknown. We have previously shown that repeated cycles of binge alcohol intake and withdrawal activate Fyn kinase in the DMS of rodents [32-34]. Interestingly, Thompson et al. showed that Fyn is required for mTORC2 activation in mesenchymal stem cells [35] raising the possibility that Fyn is upstream of mTORC2 in the DMS. Furthermore, the consequence of Fyn activation by alcohol in the DMS is the long-term facilitation of GluN2B containing NMDA receptor (NMDAR) activity (33), and binge drinking and withdrawal produce long-lasting enhancement of NMDAR function in the DMS [33]. Huang et al. reported that mTORC2 activation in hippocampal neurons is triggered by repeated tetanic stimulation, and ex vivo application of glutamate and NMDA [13]. Thus, it is plausible that the activation of the Fyn/ GluN2B axis is required for mTORC2 activation by alcohol.

The DMS is composed of dopamine receptor 1 (D1R) and D2Rexpressing MSNs [36]. Excessive alcohol consumption elicits synaptic and structural modifications in DMS D1R but not in D2R MSNs, and the inhibition of D1R but not D2R activation in the DMS attenuates alcohol consumption [19]. Furthermore, Cheng et al. recently reported that chemogenetic activation of D1 MSNs in the DMS promotes excessive alcohol drinking in mice [31]. Thus, it is highly likely that the activation of mTORC2 by alcohol consumption is restricted to D1R MSNs.

Alcohol consumption enhances F-actin formation via mTORC2 What could be the mechanism by which mTORC2 alters F-actin assembly in the DMS? We show that mTORC2 phosphorylates and activates AKT. Interestingly, the mTORC2/AKT axis has been linked to F-actin assembly. Specifically, mTORC2-dependent AKT activation leads to the phosphorylation of the actin binding protein, Girdin, which enhances F-actin assembly in mouse fertilized eggs [37]. We also show that mTORC2 phosphorylates and thus activates SGK1. In macrophages, SGK1 was shown to promote the expression of $\mathrm{Cdc} 42$, a positive regulator of actin assembly [38], and in mast cells, SGK1-dependent calcium entry regulates actin cytoskeleton and degranulation [39]. These data suggest that SGK1 may be the link that connects mTORC2 to F-actin. 
In addition to AKT and SGK1, activation of mTORC2 results in the phosphorylation of protein kinase a (PKCa) $[7,40]$. PKC isoforms have been implicated in actin polymerization by phosphorylating number of downstream substrates associated with actin filaments [40]. Unfortunately, we were unable to test PKCa activity due to the poor quality of the antibodies. Finally, mTORC2 may be directly linked to actin dynamics, as Rictor recruits Tiam1, a guanine exchange factor that promotes the activity of the actin cytoskeleton regulator, Rac1 [41]. These potential transducers of mTORC2-dependent F-actin assembly will be explored in future studies.

mTORC2 is a key regulator of alcohol-dependent structural modifications in the DMS

We previously showed that excessive alcohol drinking increases the complexity of the dendritic branches and the density of mature mushroom-shaped spines in D1R MSNs [19]. Here, we provide evidence to suggest that alcohol-dependent alterations in spine structure but not in dendritic branches are mediated by mTORC2. The lack of mTORC2 involvement in alcohol-dependent alterations in dendritic branch length and complexity suggests that these morphological changes are triggered by other mechanisms. One such mechanism may involve microtubule assembly which has been shown to transiently polymerize in dendritic spines to regulate synaptic plasticity [3, 42]. Specifically, we previously reported that alcohol consumption increases microtubule assembly in the NAc in part through the phosphorylation of GSK3 $\beta$ [43]. Therefore, it would be of interest to study the involvement of microtubule dynamics in the alcohol-dependent dendritic branching and complexity in DMS MSNs.

mTORC2 and alcohol-related behaviors

We found that knockdown of Rictor in the DMS reduces alcohol consumption, whereas intra-DMS infusion of an mTORC2 activator promotes alcohol drinking.

Alcohol intake was reduced by Rictor knockdown when measured $4 \mathrm{~h}$ but not $24 \mathrm{~h}$ after the beginning of the drinking session, suggesting that mTORC2 is important for the initiation of the alcohol binge drinking episode. This finding is in accordance with the transient alcohol-dependent phosphorylation of $\mathrm{Ser}^{473}$. AKT in the DMS, returning to basal levels after $24 \mathrm{~h}$ of withdrawal [21]. The observation that mTORC2 activation is transient suggests that the morphological changes at the dendritic spines levels are rapid and dynamic. In addition, the increase in alcohol intake in response to intra-DMS infusion of A-445634 was observed within the first hour of the drinking session, suggesting that the morphological adaptations induced by MTORC2 activation in the DMS occur, at least in part, within an hour. However, the pharmacological properties of A-445634 including a possible short half-life, and drug selectivity should be taken into consideration for the interpretation of the data. Nevertheless, further studies are needed to determine the precise time-course of the mTORC2-dependent adaptations in the DMS after excessive alcohol consumption.

Various phenotypes associated with alcohol drinking depend on learning and memory processes [44, 45]. The DMS is part of a frontal-striatal circuit that drives goal-directed behaviors, which rely heavily on learning and memory processes [20]. mTORC2 in the hippocampus has been linked to memory consolidation [13]. Therefore, our results raise an interesting possibility that alcohol-mediated activation of mTORC2 in the DMS produce molecular and structural adaptations that in turn drive the consolidation of the memory of goal-directed alcohol use.

The raw data can be found at https://doi.org/10.17632/ 5 crhwhdtzj.1. Readers can access the data by creating an account and searching for "LaguesseNPP".

\section{FUNDING}

This research was supported by the National Institute of Alcohol Abuse and Alcoholism, P50AA017072 (DR), and the Belgian American Educational Foundation (SL).

\section{ADDITIONAL INFORMATION}

Supplementary information accompanies this paper at https://doi.org/10.1038/ s41386-018-0012-1.

Conflict of interest: The authors declare that they have no conflict of interest.

\section{REFERENCES}

1. Honkura N, Matsuzaki M, Noguchi J, Ellis-Davies GC, Kasai H. The subspine organization of actin fibers regulates the structure and plasticity of dendritic spines. Neuron. 2008;57:719-29.

2. Chazeau A, Giannone G. Organization and dynamics of the actin cytoskeleton during dendritic spine morphological remodeling. Cell Mol Life Sci. 2016;73:3053-73.

3. Shirao T, Gonzalez-Billault C. Actin filaments and microtubules in dendritic spines. J Neurochem. 2013;126:155-64.

4. Rothenfluh A, Cowan CW. Emerging roles of actin cytoskeleton regulating enzymes in drug addiction: actin or reactin'? Curr Opin Neurobiol. 2013;23:507-12.

5. Yan Z, Kim E, Datta D, Lewis DA, Soderling SH. Synaptic actin dysregulation, a convergent mechanism of mental disorders? J Neurosci: Off J Soc Neurosci. 2016;36:11411-7.

6. Saxton RA, Sabatini DM. mTOR signaling in growth, metabolism, and disease. Cell. 2017;169(2):361-71.

7. Oh WJ, Jacinto E. mTOR complex 2 signaling and functions. Cell Cycle. 2011;10:2305-16.

8. Bockaert J, Marin P. mTOR in brain physiology and pathologies. Physiol Rev. 2015;95:1157-87.

9. Zinzalla V, Stracka D, Oppliger W, Hall MN. Activation of mTORC2 by association with the ribosome. Cell. 2011;144:757-68.

10. Garcia-Martinez JM, Alessi DR. mTOR complex 2 (mTORC2) controls hydrophobic motif phosphorylation and activation of serum- and glucocorticoid-induced protein kinase 1 (SGK1). Biochem J. 2008;416:375-85.

11. Sarbassov DD, Guertin DA, Ali SM, Sabatini DM. Phosphorylation and regulation of Akt/PKB by the rictor-mTOR complex. Science. 2005;307:1098-101.

12. Sarbassov DD, Ali SM, Kim DH, Guertin DA, Latek RR, Erdjument-Bromage $\mathrm{H}$, et al. Rictor, a novel binding partner of mTOR, defines a rapamycin-insensitive and raptor-independent pathway that regulates the cytoskeleton. Curr Biol. 2004;14:1296-302.

13. Huang W, Zhu PJ, Zhang S, Zhou H, Stoica L, Galiano M, et al. mTORC2 controls actin polymerization required for consolidation of long-term memory. Nat Neurosci. 2013;16:441-8.

14. Johnson JL, Huang W, Roman G, Costa-Mattioli M. TORC2: a novel target for treating age-associated memory impairment. Sci Rep. 2015;5:15193.

15. Thomanetz V, Angliker N, Cloetta D, Lustenberger RM, Schweighauser M, Oliveri $\mathrm{F}$, et al. Ablation of the mTORC2 component rictor in brain or Purkinje cells affects size and neuron morphology. J Cell Biol. 2013;201:293-308.

16. Dadalko Ol, Siuta M, Poe A, Erreger K, Matthies HJ, Niswender K, et al. mTORC2/ rictor signaling disrupts dopamine-dependent behaviors via defects in striatal dopamine neurotransmission. J Neurosci. 2015;35:8843-54.

17. Costa-Mattioli M, Monteggia LM. mTOR complexes in neurodevelopmental and neuropsychiatric disorders. Nat Neurosci. 2013;16:1537-43.

18. Mazei-Robison MS, Koo JW, Friedman AK, Lansink CS, Robison AJ, Vinish M, et al. Role for mTOR signaling and neuronal activity in morphine-induced adaptations in ventral tegmental area dopamine neurons. Neuron. 2011;72:977-90.

19. Wang J, Cheng Y, Wang X, Roltsch Hellard E, Ma T, Gil H, et al. Alcohol elicits functional and structural plasticity selectively in dopamine D1 receptor-expressing neurons of the dorsomedial striatum. J Neurosci. 2015;35:11634-43.

20. Hart G, Leung BK, Balleine BW. Dorsal and ventral streams: the distinct role of striatal subregions in the acquisition and performance of goal-directed actions. Neurobiol Learn Mem. 2014;108:104-18.

21. Laguesse $S$, Morisot N, Phamluong K, Ron D. Region specific activation of the AKT and mTORC1 pathway in response to excessive alcohol intake in rodents. Addict Biol. 2016;22:1856-69.

22. Laguesse S, Morisot N, Shin JH, Liu F, Adrover MF, Sakhai SA, et al. Prosapip1dependent synaptic adaptations in the nucleus accumbens drive alcohol intake, seeking, and reward. Neuron. 2017;96:145-59.e8. 
23. Ben Hamida S, Neasta J, Lasek AW, Kharazia V, Zou M, Carnicella S, et al. The small $\mathrm{G}$ protein $\mathrm{H}$-Ras in the mesolimbic system is a molecular gateway to alcoholseeking and excessive drinking behaviors. J Neurosci. 2012;32:15849-58.

24. Han EK, Leverson JD, McGonigal T, Shah OJ, Woods KW, Hunter T, et al. Akt inhibitor A-443654 induces rapid Akt Ser-473 phosphorylation independent of mTORC1 inhibition. Oncogene. 2007;26:5655-61.

25. Cingolani LA, Goda Y. Actin in action: the interplay between the actin cytoskeleton and synaptic efficacy. Nat Rev Neurosci. 2008;9:344-56.

26. Manning BD, Toker A. AKT/PKB signaling: navigating the network. Cell. 2017;169:381-405.

27. Kasai H, Matsuzaki M, Noguchi J, Yasumatsu N, Nakahara H. Structure-stabilityfunction relationships of dendritic spines. Trends Neurosci. 2003;26:360-8.

28. Dietz DM, Sun H, Lobo MK, Cahill ME, Chadwick B, Gao V, et al. Rac1 is essential in cocaine-induced structural plasticity of nucleus accumbens neurons. Nat Neurosci. 2012;15:891-6.

29. Li G, Wang Y, Yan M, Xu Y, Song X, Li Q, et al. Inhibition of actin polymerization in the NAc shell inhibits morphine-induced CPP by disrupting its reconsolidation. Sci Rep. 2015;5:16283.

30. Toda S, Shen HW, Peters J, Cagle S, Kalivas PW. Cocaine increases actin cycling: effects in the reinstatement model of drug seeking. J Neurosci: Off J Soc Neurosci. 2006;26:1579-87.

31. Cheng Y, Huang CCY, Ma T, Wei X, Wang $X$, Lu J, et al. Distinct synaptic strengthening of the striatal direct and indirect pathways drives alcohol consumption. Biol Psychiatry. 2017;81:918-29.

32. Gibb SL, Hamida SB, Lanfranco MF, Ron D. Ethanol-induced increase in Fyn kinase activity in the dorsomedial striatum is associated with subcellular redistribution of protein tyrosine phosphatase alpha. J Neurochem. 2011;119:879-89.

33. Wang J, Lanfranco MF, Gibb SL, Yowell QV, Carnicella S, Ron D. Long-lasting adaptations of the NR2B-containing NMDA receptors in the dorsomedial striatum play a crucial role in alcohol consumption and relapse. J Neurosci. 2010;30:10187-98.

34. Darcq E, Hamida SB, Wu S, Phamluong K, Kharazia V, Xu J, et al. Inhibition of striatal-enriched tyrosine phosphatase 61 in the dorsomedial striatum is sufficient to increased ethanol consumption. J Neurochem. 2014;129:1024-34.
35. Thompson WR, Guilluy C, Xie Z, Sen B, Brobst KE, Yen SS, et al. Mechanically activated Fyn utilizes mTORC2 to regulate RhoA and adipogenesis in mesenchymal stem cells. Stem Cells. 2013;31:2528-37.

36. Gerfen CR, Surmeier DJ. Modulation of striatal projection systems by dopamine. Annu Rev Neurosci. 2011;34:441-66.

37. Enomoto A, Murakami $\mathrm{H}$, Asai $\mathrm{N}$, Morone $\mathrm{N}$, Watanabe $\mathrm{T}$, Kawai $\mathrm{K}$, et al. Akt/PKB regulates actin organization and cell motility via Girdin/APE. Dev Cell. 2005;9:389-402.

38. Ding L, Zhang L, Kim M, Byzova T, Podrez E. Akt3 kinase suppresses pinocytosis of low-density lipoprotein by macrophages via a novel WNK/SGK1/Cdc42 protein pathway. J Biol Chem. 2017;292:9283-93.

39. Schmid E, Gu S, Yang W, Munzer P, Schaller M, Lang F, et al. Serum- and glucocorticoid-inducible kinase SGK1 regulates reorganization of actin cytoskeleton in mast cells upon degranulation. Am J Physiol Cell Physiol. 2013;304: c49-55.

40. Larsson C. Protein kinase $C$ and the regulation of the actin cytoskeleton. Cell Signal. 2006;18:276-84.

41. Tejada-Simon MV. Modulation of actin dynamics by Rac1 to target cognitive function. J Neurochem. 2015;133:767-79.

42. Dent EW. Of microtubules and memory: implications for microtubule dynamics in dendrites and spines. Mol Biol Cell. 2017;28:1-8.

43. Liu F, Laguesse S, Legastelois R, Morisot N, Ben Hamida S, Ron D. mTORC1dependent translation of collapsin response mediator protein-2 drives neuroadaptations underlying excessive alcohol-drinking behaviors. Mol Psychiatry. 2017;22:89-101.

44. Barak S, Liu F, Ben Hamida S, Yowell QV, Neasta J, Kharazia V, et al. Disruption of alcohol-related memories by mTORC1 inhibition prevents relapse. Nat Neurosci. 2013;16:1111-7.

45. Goodman J, Packard MG. Memory systems and the addicted brain. Front Psychiatry. 2016;7:24.

46. Ron D, Barak S (2016). Molecular mechanisms underlying alcohol-drinking behaviours. Nat Rev Neurosci 17(9): 576-591. 